\title{
A LOOK ON NON-STANDARD SOLAR MODELS
}

\author{
André Maeder \\ Geneva Observatory \\ CH-1290 Sauverny, Switzerland
}

\begin{abstract}
A short review of some of the non-standard models proposed in these last two decades is presented. Their main physical assumptions are shown, as well as the way they meet or do not meet the various observational constraints.
\end{abstract}

\section{PHILOSOPHY OF NON-STANDARD MODELS}

The French physicist J.M. Levy-Leblond (1981) in a paper entitled "Eloge des theories fausses" has distinguished four kinds of false theories: 1. The adherent ones, which are correct over a sizeable but still limited range of conditions. 2 . The different ones, which are internally coherent but finally in disagreement with nature. 3 . The aberrant ones, which contain big mistakes and errors. 4. The "siderant" ones, which are just scientific phrasing without scientific meaning. Of course, the non-standard models considered here do not belong to the last two categories! Apart from joke and as emphasized by Levy-Leblond, it is very didactical to understand why a non-standard theory may fit or not; a better comprehension of the physics is gained in this way.

Numerous non-standard solar models have been proposed, mainly stimulated by the solar neutrinos problems. They cannot all be right, but anyhow the study of their properties and why they fit or do not fit the observational requirements gives us a deeper insight and understanding of solar and stellar physics. Also, it is quite likely that some features of today's non-standard models will be retained in the standard models of tomorrow.

On the whole we must say that the study of many non-standard models has also increased our confidence in the standard model (cf. Bahcall, 1989). Some deviation from it will probably be found and confirmed in the future, but it is unlikely to be large.

Among other recent reviews on non-standard models we may quote that by Newman (1986) and the one by Bahcall and Ulrich (1988).

\section{MODELS WITH ADDITIONAL EFFECTS IN CLASSICAL PHYSICS}

This is the broadest and, in my opinion, most interesting class of non-standard models. Some of their properties will certainly be incorporated as basic ingredients in future, particularly regarding transport processes and rotation.

\subsection{Models with transport in absence of rotation}

Thermal diffusion and gravitational settling is just standard physics. These processes have been included in the solar model by Gabriel et al. (1983), and more recently by Cox et 
al. (1989), using a procedure devised by Iben and McDonald (1985). Cox et al. find that the helium and metal abundances changed from $Y=0.30$ to 0.263 , and from $Z=.02$ to .179 at the solar surface during its past evolution. At the center the effects are of the order of $10^{-3}$. Clearly, no major change occurs neither for the solar neutrino flux, nor for the p-mode frequencies which are still too small by $10-20 \mu \mathrm{Hz}$. Anyhow, the effects on surface abundances and opacities are significant.

The possibility that the positive He-3 gradient produces instabilities leading to mixing was examined by Dilke and Gough (1972); cf. also Ulrich and Rood (1973). According to Christensen-Dalsgaard et al. (1974), the He-3 gradient may produce unstable low order $\mathrm{g}-$-modes, but the issue regarding mixing remains uncertain.

The case of nuclear instabilities in the Sun was studied with negative conclusions by Schwarzschild and Härm (1973) and Gilliland (1985) for the radial case and by Rosenbluth and Bahcall (1973) in the non radial case.

\subsection{Models with rotational mixing}

A high interior ratio of centrifugal force to gravity may lower the central $T$ and thus the neutrino flux. However, the data on solar oblateness place some constraints (cf. Rood and Ulrich, 1974) on the many suggestions made along that line in the past. Rotationally induced mixing is certainly a more promising possibility in which rotation could influence solar evolution.

\subsubsection{Models with rotationally induced turbulent diffusion}

The concept of turbulent diffusion was introduced by Schatzman (1977) and applied in a series of papers by himself and coworkers. The idea is the following one. According to Schatzman (1962) the slow rotation of late type stars results from the loss of angular momentum by stellar winds. This leads to differential rotation, the interior spinning faster than the surface. The increase of the angular velocity with depth is limited by the hydrodynamical instabilities, which cause an outward diffusion of angular momentum. The external convective zone of the Sun is highly viscous and rotates nearly at the surface rate. Schatzman (1977) suggested that the shear between the rapidly rotating interior and the external convective zone is unstable and leads to turbulence. The angular momentum is transported from core to surface, therefore reducing the differential rotation rate until stability is restored. Further loss of angular momentum again generates a shear flow with a large angular velocity gradient, thus re-establishing the turbulent regime. Schatzman suggests that the Sun adjusts itself in such a way that it is always locally at the edge of shear flow instability. As it is difficult to predict the level of the turbulence and its transport efficiency, he considers turbulent mixing as an isotropic diffusion process, the effective diffusivity $D$ being larger than the microscopic viscosity by a constant factor called Re*. The diffusion equation is:

$$
\rho \frac{\partial X_{i}}{\partial t}=\operatorname{div}\left(\rho D \operatorname{grad} X_{i}\right)
$$

with

$$
D=\operatorname{Re}^{*}\left(\nu_{\mathrm{rad}}+\nu_{\mathrm{mol}}\right)
$$

where $\nu_{\text {rad }}$ and $\nu_{\text {mol }}$ are respectively the radiative and molecular viscosities.

Since these first tentative investigations much progress has been made in the understand. ing of the instabilities and their occurrence in rotating stars (Zahn, 1983, 1985; Spruit, 1985; 
cf. also Tassoul and Tassoul, 1989). Zahn proposes the following very interesting mixing mechanism: when a star does not rotate cylindrically, the surfaces of constant pressure and the isentropic ones no longer coincide and the equilibrium state is called baroclinic; in the very small wedge between a surface of constant entropy and the corresponding horizontal surface horizontal motions may become unstable, generating turbulence. The turbulence is two-dimensional, at least for scales where the Coriolis force is greater than the inertial one (i.e. for scales which satisfy the Rossby criterion: $\Omega>2.5 u / l$ ). The usual cascade of turbulence towards small scales leads to a three-dimensional turbulence as soon as the Rossby condition is violated. This is this small scale 3-D tail of the 2-D turbulence which is responsible, according to $\mathrm{Zahn}$, for the vertical diffusion of the angular momentum and chemical elements. Assuming that the kinetic energy of differential rotation, fed itself by advection of the angular momentum, is converted into turbulent energy, Zahn obtains the following value of the turbulent diffusion coefficient $D$, due to the 3-D turbulence:

$$
D=\operatorname{Re}^{*} \nu
$$

with

$$
\operatorname{Re}^{*} \simeq \frac{K}{\nu} \frac{\Omega^{2} r}{g}\left(\nabla_{\text {ad }}-\nabla_{\text {rad }}\right)^{-1}
$$

where $r$ is the distance to the center, $g$ the gravity at distance $r, \Omega$ the angular velocity, $\nabla_{\text {ad }}-\nabla_{\text {rad }}$ the local subadiabatic gradient in the radiative zone and $K$ is the thermal diffusivity. This holds in the local instability approximation (i.e. it is not valid near the center). The simulation of models with this $\mathrm{Re}^{*}$-expression is difficult, because we do not know the spatial distribution of the differential rotation $\Omega$ in the Sun and other main sequence stars. The value of $\Omega$ should depend on the radius $(r)$ and on the latitude $(\Lambda)$ considered. However, Zahn (1973) has shown that an $\Omega$-dependence on $\Lambda$ would imply a rapid re-distribution of angular momentum along equipotential surfaces by finite amplitude shear instabilities, so as to make $\Omega$ constant on such surfaces. This prediction was beautifully confirmed by the analysis of the frequency splitting of solar $p$-models (cf. Brown, 1985), which shows that, for growing depths, the solar latitudinal differential rotation is much smaller than at the surface, the rotation being roughly constant on spheres with a rotation rate close to the surface equatorial value. Zahn (1983) also showed that the vertical $\mu-$ gradient can prevent the 2-D turbulence from becoming 3-D, and this effect was generally accounted for in the models.

Various applications of the turbulent diffusion model were made to account for the abundances of $\mathrm{Li}, \mathrm{Be}$ and $\mathrm{B}$ at the surface of the Sun (Vauclair et al., 1978), the solar neutrino flux (Schatzman and Maeder, 1981), the ratio $\left({ }^{12} \mathrm{C} /{ }^{13} \mathrm{C}\right)$ at the surface of red giant stars (Genova and Schatzman, 1979; Bienaymé et al., 1984), the solar and stellar Liabundances (Baglin et al., 1985), the He-3 abundance and the frequencies of solar oscillations (cf. Lebreton and Maeder, 1987).

Presently, as strong constraints on the possibility of mixing in the Sun, we may mention the following ones: 
${ }^{3} \mathrm{He}:$

Bochsler et al. (this meeting; see also Geiss, 1972) show that the abundance of ${ }^{3} \mathrm{He}$ at the solar surface cannot have increased by more than $15 \%$ over the solar lifetime. This beautiful result places a very strong constraint on mixing processes in the deep interior. Indeed, as shown by Bochsler et al. the Reynolds number $\mathrm{Re}^{*}$ cannot be larger than about 30 in the interior. Equivalently, if

$$
R e^{*}=\frac{8 r}{\alpha^{2} H_{p}}
$$

as it was represented by Lebreton and Maeder (1987), $\alpha \simeq 1.3$, leading to diffusion coefficients $146,225,321,538,853 \mathrm{~cm}^{2} / \mathrm{sec}$ at $M r / M=$ $.77, .85, .90, .95, .98$. Also, in this model of the Sun, the $\mu$-gradient would have stopped mixing in regions interior to $M_{r} / M=0.76$.

${ }^{7} \mathrm{Li}$ : Baglin et al. (1985) show that it is necessary to call for a diffusion process in the outer layers to account for the smooth decrease of the Li-abundance for stars of decreasing masses in the Hyades. Thus, contrarily to a frequently made assumption, the overshooting below the external convective zone is not an appropriate mechanism for explaining the low $\mathrm{Li}$ abundance in the Sun, because one would then have to call for very different overshooting parameters for stars of different masses. On the contrary, turbulent diffusion with a unique parameter is shown to be able to account for the $\mathrm{Li}$ abundances in stars of various masses.

Solar p-modes: In order to explain by turbulent diffusion the low solar neutrino flux, it would be necessary to assume a rather high diffusion coefficient of the order of $\mathrm{Re}^{*}=100$ (cf. Schatzman and Maeder, 1981). However, a number of authors have shown that such a high diffusion would be in contradiction with the results on the low degree solar acoustic modes (cf. Ulrich and Rhodes, 1983; Provost, 1984; Berthomieu et al., 1984; Cox et al., 1985; Christensen-Dalsgaard, 1986; Lebreton and Maeder, 1987; Lebreton et al., 1988). In particular, the splitting of low degree modes is much too large in models with a diffusion. However, as shown by Lebreton and Maeder, diffusion with a moderate coefficient $\mathrm{Re}^{*}$ and with inclusion of the $\mu$-gradient inhibition does not change the splitting values.

Figure 1 shows the difference $\delta c^{2} / c^{2}$ betwen a strongly mixed $\left(\operatorname{Re}^{*}=\right.$ 100) and a standard model (cf. Christensen-Dalsgaard, 1986) where $c$ is the sound velocity. As $c \sim(T / \mu)^{1 / 2}$, the high central peak is due to the lower $\mu$ of the diffusion model, which is compensated by a higher $\mu$ and a lower $c$ outwards from $r / R=0.15$. It is interesting to notice that the shape in Fig. 1 is very reminiscent, but with a lower amplitude, of the difference between the observed and the standard model (cf. Gough and Kosovichev, 1988). 


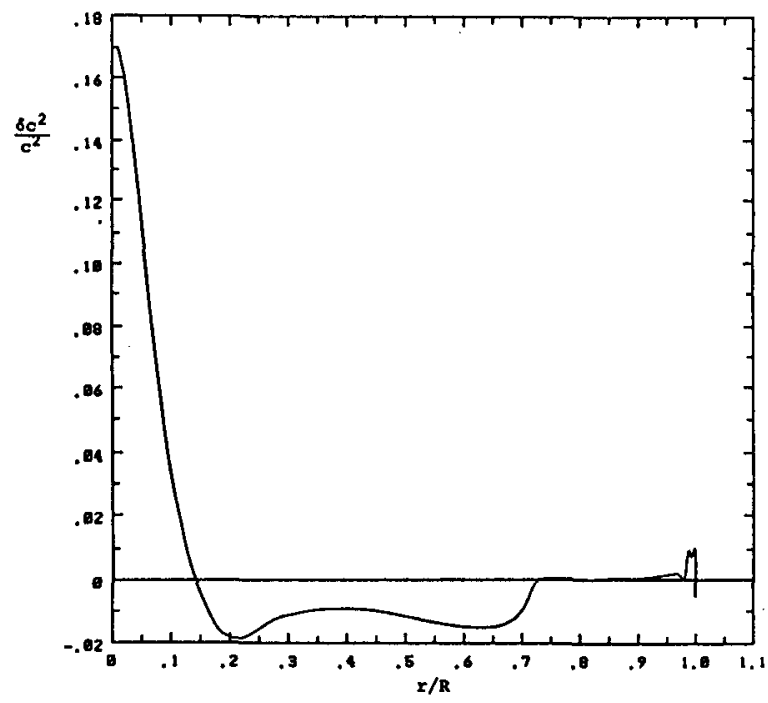

Figure 1: Difference $\delta c^{2} / c^{2}$ between a strongly mixed $\left(R e^{*}=100\right)$ and a standard model, $c$ being the sound velocity (from Christensen-Dalsgaard, 1986).

Solar g-modes: Mixing leads to flatter $\mu$ and temperature gradients in the Sun. Thus, the restoring force in radiative regions is smaller and so is the BruntVäisälä frequency. Thus, the period spacing $P_{o}$ of the gravity modes in partially mixed models is increased with respect to the standard case (cf. Berthomieu et al., 1984; Cox et al., 1985; Lebreton and Maeder, 1987). The observed values of $P o$ lie in the range of 36 to 41 minutes, while standard models give values of $P o$ between 34 and 37 minutes. This discrepancy is compatible with a value $\mathrm{Re}^{*}$ of about 20 in deep interior regions $(r / R \simeq 0.15)$.

Thus, it is very interesting to notice that the $\mathrm{He}-3$ abundance and solar oscillations place the same rather severe limit on diffusive mixing in the deep interior. However, it is important to realize that if the mixing would effectively be at the limit of $\mathrm{Re}^{*}=20$ or 30 , very significant consequences would result for the evolution of the Sun and low mass stars. In particular, the lifetimes of the low mass stars would be increased, with non-negligible consequences for the age determinations.

\subsubsection{Models with rotational mixing and transport of angular momentum}

The rotational velocity and the internal distribution of the angular velocity $\Omega(r)$ in the Sun starts becoming basic observational constraints to be added to the traditional $\mathrm{M}, \mathrm{L}, \mathrm{R}, \mathrm{T}_{\text {eff }}$ abundances. Since long valuable attempts to properly modelize the internal $\Omega(r)$ have been made (cf. Endal, 1987; Sofia, 1987; and reference therein). Recently Pinsonneault et al. (1989) have developed a new rotating stellar evolution code and applied it to the Sun. As specific basic ingredients they incorporate the loss of angular momentum $J$ via magnetic 
winds by adopting some parametrisation of $d J / d t$ in terms of $\Omega, M, R$ and mass loss rate $\dot{M}$. The redistribution of angular momentum by various processes, such as Eddington circulation, Goldreich-Schubert-Fricke instability and shear instability, is accounted for; the mixing of chemical species resulting from these instabilities and circulation motions are also considered. This modellisation of complex processes of course contains free parameters that the authors adjust on the observations.

These models of the rotating Sun contain several very interesting results among which we may mention:

- In order to reproduce both the observations or abundances and rotation, the diffusion coefficient of chemical elements has to be only a small fraction (i.e. about $5 \%$ ) of the diffusion coefficient of the angular momentum (cf. Spruit, 1987; Schatzman, 1987; see also Tassoul and Tassoul, 1989).

- The angular velocity $\Omega$ is nearly constant in the outer layers $(r / R>0.5)$. A central fast spinning core (with rotation period $<12 h$ ) is present and these features are found for a wide range of global parameters.

- The present $\Omega$ at the surface of low mass stars is weakly dependent on the initial angular momentum. The reason is that for fast rotation the loss of angular momentum at the surface is also higher, as well as the redistribution process.

- However, the value of the initial angular momentum strongly influences the present values of the abundances of some elements, in particular $\mathrm{Li}$ and to a lesser extent $\mathrm{Be}$ and $\mathrm{He}-3$ in the Sun. The reason is that for a higher initial angular momentum, the instabilities and transport processes are also more active. Rotation also influences CNO abundances in red giants after the first dredge-up. The necessity of an additional main sequence destruction of lithium in order to explain the observed $\mathrm{Li}$ abundances in red giants has also been shown recently by Charbonneau et al. (1989).

Recently, Tassoul and Tassoul (1989) have calculated the secular changes of the solar inner rotation, taking into account the solar wind torque as well as the gradual reduction of the eddy diffusivities in the chemically inhomogeneous inner regions. In particular, they show that a moderate amount of eddy-like or wave-like motions is sufficient to keep the inner and outer parts of the Sun's radiative interior rotating nearly uniformly. Thus, there is no reason, according to Tassoul and Tassoul, to assume the existence of a large magnetic field that could enforce the uniform rotation by itself. They also suggest a ratio of eddy diffusivity to eddy viscosity of the order of 5 to $10 \%$, as quoted above.

\subsection{Models with magnetic fields}

The inclusion of magnetic pressure $B^{2} / 8 \pi$ could in principle lower the central temperature. But a proper corresponding balance of the helium and metal contents has to be made and then, as shown by Iben (1969) and Bahcall and Ulrich (1971), there is no longer a reduction of central $T$ and neutrino flux to be expected.

The magnetic tension term $B \cdot(\nabla B)$ has to be included and this requires a modellisation of the field geometry. Models with a magnetic field of the Cowling type were made by Ulrich and Rhodes (1983). They showed that the tension term leads to a reduction of central pressure and neutrino flux. However, the field has to be very high, i.e. $3 \cdot 10^{8}$ 
Gauss for a decrease of the neutrino flux of $30 \%$. An attractive feature is that the p-mode frequencies, and spacing as well, are increased in the sense required by observations.

\subsection{Models with accretion or high mass loss}

Accretion was topical for a time with the suggestion by McCrea (1975) that ice ages on the earth may be due to the entering of the solar system into a dense interstellar cloud. Both the effects of extinction and of the release of gravitational energy should be accounted for. Current estimates of the mass accreted by the Sun in its evolution give (cf. Talbot and Newman, 1976) values of the order of $10^{-4} M_{\odot}$. Thus, this effect is unlikely to have had great consequences.

The idea that the Sun might have experienced a relatively high mass loss was put forward by Willson et al. (1987) and Guzik et al. (1987). They suggest that main-sequence stars located in the pulsation instability strip may experience mass loss rates in excess of $10^{-9} M_{\odot} y^{-1}$. Thus typically, starting on the zero age sequence with an initial mass of about $2 M_{\odot}$, the Sun would reach its present mass in about $10^{9} y$. There is neither detailed physical process demonstrated to be able to do that, nor any direct observational confirmation of the advocated mass loss rates. The authors propose this high mass loss to be responsible for the existence of blue stragglers; the consequences for the age determination and history of the Galaxy are also emphasized.

A fortunate and unvoluntary consequence of this model is to demonstrate how powerful and constraining some of the available observational data are.

$-{ }^{3} \mathrm{He}$ : The new and reliable determination of the ${ }^{3} \mathrm{He}$ abundance at the solar surface by Bochsler et al. (1989) shows that the ${ }^{3} \mathrm{He}$ enrichment cannot be larger than $15 \%$ during the solar life, while the models by Guzik et al. predict an enrichment of nearly an order of magnitude. There is no way to circumvent this difficulty.

- Li, Be, B: These elements would have been very early completely destroyed at the solar surface in the model of the mass-losing Sun. Guzik et al. (1987) advocate for spallation reactions to synthesize these elements at their present level in the Sun. Indeed, if this were true, the $\mathrm{Li}$ abundance of low mass stars in clusters would increase with cluster ages. The observations by Hobbs and Pilachowski (1988; see also Twarog and Twarog, 1989) show just the opposite: Li regularly declines for old age clusters. Moreover, the model by Guzik et al. predict complete destruction of $\mathrm{Li}$ in the solar-like stars of the Pleiades, while the observations on the contrary show an absence of depletion with respect to the interstellar medium.

- Solar oscillations: The course of the sound speed in the interior of a solar model having experienced a high mass loss is incompatible as shown by Turck-Chieze et al. (1988) with the helio-seismological results. The limit placed by the inversion technique on the total amount of mass lost by the Sun is about $0.2 M_{\odot}$. This is much less constraining than the Li-data in clusters, which tell us that the Sun may not have lost more than about $3 \%$ of its mass during the first hundreds of million years. Otherwise Li would already be entirely depleted in clusters in the age range from Pleiades to Hyades, which is not the case.

In conclusion, three different valuable observations let no room for a highly mass-losing solar model.

\subsection{Models with effects resulting from pre-main sequence evolution}

Some effects in pre-main sequence evolution, such as a separation of the elements in the solar nebula, could still influence the present structure of the Sun. We may range the 
inhomogeneous initial solar models in this category, although their authors frequently just aimed at providing some trial and test calculations, which often are very instructive.

A low interior metallicity $Z$ (with a proper account of the helium abundance in order to match the solar luminosity) leads to a lower solar neutrino flux (e.g. Iben, 1969; Ulrich and Rhodes, 1983; Bahcall and Ulrich, 1988). However, as shown for example by these last authors (cf. also Christensen-Dalsgaard and Gough, 1980; Ulrich and Rhodes, 1983), the low $Z$ model increases the disagreement between the observed and calculated frequency of low degree $\mathrm{p}$-modes.

The consequences of a high interior helium content $Y$ were also explored (e.g. Iben, 1969; e.g. Bahcall and Ulrich, 1988). The following interesting result was shown by Bahcall and Ulrich. Some changes of the Sun's composition, for example an increase in $Y$ by 0.025 between $\mathrm{Mr} / M_{\odot}=0.2$ to 0.4 , can considerably improve the agreement for solar oscillations with little change for the neutrino flux, which illustrates the great specificity of the various constraints.

Another consequence of pre-main sequence evolution concerns the possibility of survival of convective cores in the Sun. It was occasionally suggested that a central convective core may be sustained for some time by convective overshooting (e.g. Roxburgh, 1987). In this connection, it would be quite interesting to examine how the presence and temporary survival of a convective core at the beginning of main sequence evolution depend on the proper or improper equilibration of $\mathrm{He}-3$ and $\mathrm{CN}$ elements as a result of pre-main sequence evolution.

\section{EXOTIC MODELS}

Such models either call for "new" physics, not (or maybe not yet) generally accepted or not confirmed by experience or, as for the model of the Sun with a central black hole, they bring a well accepted concept in a context where it is highly unusual. However, it is very hazardous to attempt to further characterize these models. Perhaps the only common feature to all these exotic models is that they generally have been proposed with a very specific aim in mind (e.g. solving the neutrino problem) and that the authors have not submitted their models to the widest range of possible observational tests.

\subsection{Models with modified gravity}

Many solar models in the line of Dirac's Large Number Hyphthesis were made (see for example Pochoda and Schwarzschild, 1964; Maeder, 1977). In the case proposed by Dirac, where the gravity constant $G$ varies like $1 / t$, where $t$ is the cosmic time, the Sun would have been more tightly packed together in the past and would look more evolved. The explanation of the present solar luminosity would require a much too low initial He-content. Other cases were considered in the framework of the scale covariant cosmology (cf. Canuto et al., 1977; see also Maeder, 1979), but the solar tests were generally less constraining than astrometric measurements in the solar system, which generally do not favour such theories.

Central gravity in stars could also be modified by the hypothetic presence of central black holes remaining from the Big Bang (cf. Hawking, 1971). Clayton et al. (1975b) considered black holes of $10^{-5} M_{\odot}$, assumed to radiate at their Eddington luminosity. The auxiliary energy source provides about half of the solar luminosity and the central temperature is thus reduced, which leads to a low solar neutrino flux. The various implications of this model in other evolutionary phases (e.g. star formation) have not been worked out. 
The effects of the so-called fifth force, which would reduce the gravity constant $G$ over short ranges ( $\$ 200 \mathrm{~m}$ ), have been examined by Gilliland and Däppen (1987) for their consequences on solar and stellar evolution. They find that the changes to solar structure, neutrino fluxes and oscillation frequencies are within current observational and theoretical uncertainties. Stellar lifetimes could, however, undergo significant changes.

\subsection{Non standard effects affecting nuclear physics}

There are so many explicit or implicit hypotheses made in stellar microphysics, which are generally no longer questioned and are adopted once for ever, that any critical discussion in this context is most welcome. Such is the case for the study by Clayton et al. (1975a) of possible depletion of the high energy tail of the Maxwell distribution. Even a very weak depletion could highly reduce the solar neutrino flux, and Clayton's et al. work illustrated the need for many body physics in the study of long-range Coulomb interactions.

More exotic was the proposal by Libby and Thomas (1969) of fusion catalysis by free quarks which, even if present in stellar interiors, would not appreciably affect the energy production in the Sun (cf. Salpeter, 1970).

The question of hypothetic small violations of Pauli Principle was raised by Plaga, (1989).

Very valuable exploratory calculations were performed by Bahcall and Ulrich (1988) who examined the consequences of non-standard nuclear physics on solar structure, solar oscillations and neutrino flux, by arbitrarily modifying the rates of some nuclear reactions. Such simulations allowed them to enlighten the most critical factors for the various reactions considered.

\subsection{Modified energy transport}

The consequences of changes in the process of energy transport have been studied by Newmann and Fowler (1976): more transport means a lower $T$-gradient and thus a lower neutrino flux. More recently Faulkner and Swenson (1988) examined the consequences of the existence of isothermal core in stars, by arbitrarily multiplying the opacity coefficient by a factor $10^{-3}$ in the central $5 \%$ of the solar mass. They found that stellar evolution on the main sequence is then determined by the attempt of violation of the SchönbergChandrasekhar limiting mass fraction. These studies were made in relation with the WIMP models, a presently interesting and topical class of non-standard models into which we shall not enter here since it is largely covered by other presentations at this meeting. These models assume exotic particles, the WIMPS, affecting the energy transport in stars. Massive non-baryonic particles were also considered for their effects as internal energy sink by Finzi and Harpaz (1989).

\section{CONCLUSIONS}

To the major question "Do we need to change the standard model?" one would tend to answer "yes probably, but not by very much". In the case of extra-mixing, for example, one has learned that whatever mixing may be present in the solar interior, it is not likely to be larger than as given by $R e^{*}=20-30$ in the deep interior which places rather severe limits on the motion of chemical species in the profound solar interior. On the whole, it is impressive how robust the standard model has been found over the last twenty years, despite its very simple recipes about convection and energy transport. 
Nowadays, solar and stellar evolution is fortunately subject to a larger number of observational tests, which at the same time are more accurate than those done some 30 years ago. Let us mention:

1. Values of $L_{\odot}, R_{\odot}$ at given mass and age.

2. Oscillation data on acoustic modes.

3. Oscillation data on low gravity modes.

4. Account for surface angular velocity $\Omega$, in relation with oscillation data on the angular velocity $\Omega(r)$ in the solar interior (cf. Brown, 1985).

5. Solar oblateness (cf. Hill, Stebbins, 1975).

6. Solar depletion in Li by a factor of 200 (cf. Muller et al., 1975).

7. Solar depletion in ${ }^{8}$ Be by a factor 2 to 5 (cf. Molaro and Beckman, 1984).

8. Maximum enrichment in ${ }^{3} \mathrm{He}$ of $15 \%$ during the solar life (cf. Bochsler et al., 1989).

9. Maximum increase of ${ }^{13} \mathrm{C} /{ }^{12} \mathrm{C}$ of $7 \%$ during the solar life (cf. Harris et al., 1987).

10. Solar neutrino flux (cf. Bahcall, 1989).

11. Solar magnetic field.

Related stellar data:

12. Sequences of old clusters in the HR diagram; both the shape of the sequence and the star frequency along the sequences have to be examined.

13. Distribution of rotational velocities of main sequence stars and red giants in old clusters.

14. Abundance ratios of $\mathrm{C} / \mathrm{N},{ }^{13} \mathrm{C} /{ }^{12} \mathrm{C}$ of red giants in old clusters (cf. Brown, 1987; Lambert and Ries, 1981).

15. Li in red giants of old clusters (cf. Charbonneau and Michaud, 1989).

To my knowledge, none of the non standard models have been submitted to all these tests. We would even say that, in the case of the standard models also, these tests have neither been all considered simultaneously. Ideally, they should all be performed before a new solar model can be accepted. These observational data play an essential role with respect to futur progress in stellar evolution, either as censors of theory or as guides for future research. 


\section{REFERENCES}

Baglin A., Morel P., Schatzman E.: 1985, Astron. Astrophys. 149, 309

Bahcall J.N.: 1989, these Proceedings

Bahcall J.N., Ulrich R.K.: 1971, Astrophys. J. 170, 593

Bahcall J.N., Ulrich R.K.: 1988, in Rev. Modern Physics 60, 297

Berthomieu G., Provost J., Schatzman E.: 1984, Nature 308, 254

Bienaymé O., Maeder A., Schatzman E.: 1984, Astron. Astrophys. 131, 316

Bochsler P., Geiss J., Maeder A.: 1989, these Proceedings

Brown J.A.: 1987, Astrophys. J. 317, 701

Brown T.M.: 1985, Nature 317, 591

Canuto V., Adams P.J., Hsieh S.H., Tsiang E.: 1977, Phys. Rev. D16, 1643

Charbonneau P., Michaud G., Proffitt C.R.: 1989, Astrophys. J., in press

Christensen-Dalsgaard J.: 1986, in Seismology of the Sun and the Distant Stars, Ed. D.O. Gough, Reidel Publ. Co., p. 23

Christensen-Dalsgaard J.: 1988, Proc. Symp. Seismology of the Sun and Sun-like Stars, ESA SP-286, p. 431

Christensen-Dalsgaard J., Dilke F.W.W., Gough D.O.: 1974, Monthly Notices Roy. Astron. Soc. 169,429

Christensen-Dalsgaard J., Gough D.O.: 1980, Nature 288, 545

Clayton D.D., Dwek E., Newman M.J., Talbot R.J.: 1975a, Astrophys. J. 199, 494

Clayton D.D., Newman M.J., Talbot R.J.: 1975b, Astrophys. J. 201, 489

Cox A.N., Guzik J.A., Kidman R.B.: 1989, Astrophys. J., in press

Cox A.N., Kidman R.B., Newman M.J.: 1985, in Solar neutrinos and neutrino Astronomy,

Ed. M.L. Cherry et al., American Institute of Physics, p. 93

Dilke F.W.W., Gough D.: 1972, Nature 240, 262, 293

Endal A.S.: 1987, in The internal solar angular velocity, Ed. B.R. Durney, S. Sofia, Reidel Publ. Co., p. 131

Faulkner J., Swenson F.J.: 1988, Astrophys. J. 329, L47

Finzi A., Harpaz A.: 1989, these Proceedings

Gabriel M., Noels A., Scuflaire R.: 1983, in Europhysics Study Conference on Oscillations as a probe of the Sun's interior, Catania

Geiss J.: 1972, Solar Wind, Proc. ASILOMAR Conf., NASA SP-308, 559

Genova F., Schatzman E.: 1979, Astron. Astrophys. 78, 323

Gilliland R.L.: 1985, Astrophys. J. 290, 344

Gilliland R.L., Däppen W.: 1987, Astrophys. J. 313, 429

Gough D.O., Kosovichev A.G.: 1988, Proc. Symp. Seismology of the Sun and Sun-like Stars, ESA SP-286, p. 195

Guzik J.A., Willson L.A., Brunish W.M.: 1987, Astrophys. J. 319, 957

Harris M.J., Lambert D.L., Goldman A.: 1987, Monthly Not. Roy. Astron. Soc. 224, 237

Hawking S.W.: 1971, Monthly Not. Roy. Astron. Soc. 152, 75

Hill H.A., Stebbins R.T.: 1975, Astrophys. J. 200, 471

Hobbs L.M., Pilachowski C.: 1988, Astrophys. J. 334, 734

Iben I.: 1969, Ann. Phys. 54, 164

Iben I., MacDonald J.: 1985, Astrophys. J. 296, 540

Lambert D.L., Ries L.M.: 1981, Astrophys. J. 248, 228

Lebreton Y., Berthomieu G., Provcst J.: 1988, in IAU Symp. 123, 95 
Lebreton Y., Maeder A.: 1987, Astron. Astrophys. 175, 99

Levy-Leblond J.M.: 1981, L'esprit de sel, Ed. Fayard

Libby L.M., Thomas F.J.: 1969, Nature 222, 1238

Maeder A.: 1977, Astron. Astrophys. 56, 359

Maeder A.: 1979, in Physical Cosmology, Ed. R. Balian et al., North Holland Publ. Co., 1980 , p. 534

McCrea W.H.: 1975, Nature 255, 607

Molaro P., Beckmann J.: 1984, Astron. Astrophys. 139, 394

Müller E.A., Peytremann E., de la Reza R.: 1975, Solar Physics 41, 53

Newman M.J.: 1986, in Physics of the Sun, Ed. P.A. Sturrock, Reidel Publ. Co., p. 33

Newman M.J., Fowler W.A.: 1976, Astrophys. J. 207, 601

Pinsonneault M.H., Kawaler S.D., Sofia S., Demarque P.: 1989, Astrophys. J. 338, 424

Plaga R.: 1989, these Proceedings

Pochoda P., Schwarzschild M.: 1964, Astrophys. J. 139, 587

Provost J.: 1984, in IAU Symp. 105, p. 47

Rood R.T., Ulrich R.K.: 1974, Nature 252, 366

Rosenbluth M.N., Bahcall J.: 1973, Astrophys. J. 184, 9

Roxburgh I.W.: 1987, in The internal solar angular velocity, Ed. B.R. Durney, S. Sofia, Reidel Publ. Co., p. 1

Salpeter E.E.: 1970, Nature 225, 165

Schatzman E.: 1962, Ann. Astrophys. 25, 18

Schatzman E.: 1969, Astrophys. Lett. 3, 331

Schatzman E.: 1977, Astron. Astrophys. 56, 211

Schatzman E.: 1987, in The internal solar angular velocity, Ed. B.R. Durney, S. Sofia, Reidel Publ. Co., p. 159

Schatzman E., Maeder A.: 1981, Astron. Astrophys. 96, 1

Schwarzschild M., Härm R.: 1973, Astrophys. J. 184, 5

Sofia S.: 1987, in The internal solar angular velocity, Ed. B.R. Durney, S. Sofia, Reidel Publ. Co., p. 173

Spruit H.C.: 1985, in The internal solar angular velocity, Ed. B.R. Durney, S. Sofia, Reidel Publ. Co., p. 185

Talbot R.J., Newmann M.J.: 1976, Astrophys. J. Suppl. 34, 295

Tassoul, J.-L., Tassoul, M.: 1989, Astron. Astrophys. 213, 397

Turck-Chieze S., Däppen W., Cassé M.: 1988, Proc. Symp. Seismology of the Sun and Sun-like Stars, ESA SP-286, p. 629

Twarog B.A., Anthony-Twarog B.J.: 1989, Astron. J. 97, 759

Ulrich R.K., Rhodes J.: 1983, Astrophys. J. 265, 551

Ulrich R.K., Rood R.T.: 1973, Nature Phys. Sci. 241, 111

Vauclair S., Vauclair G., Schatzman E., Michaud G.: 1978, Astrophys. J. 223, 567

Willson L.A., Bowen G.H., Struck-Marcell C.: 1987, Comments Astrophys. 12, 17

Zahn J.P.: 1973, in Stellar Stability and Evolution, Eds. P. Ledoux, A. Noels, A.W. Rogers, Reidel Publ. Co., 1974, p. 185

Zahn J.P.: 1983, in Astrophysical Processes in Upper Main Sequence Stars, 13th Advanced Course Saas-Fee, Publ. Geneva Observatory

Zahn J.P.: 1985, in The internal solar angular velocity, Ed. B.R. Durney, S. Sofia, Reidel Publ. Co., p. 201 